\title{
Nucleoside Reverse Transcriptase Inhibitor
}

National Cancer Institute

\section{Source}

National Cancer Institute. Nucleoside Reverse Transcriptase Inhibitor. NCI Thesaurus.

Code 197452.

A substance with a structure similar to a nucleoside molecule that is capable of occupying the active catalytic site of a reverse transcriptase resulting in inhibition of enzyme function, chain termination, and inhibition of viral replication. These compounds are converted to their active phosphorylated forms by cellular kinases. 